VERSION SEPTEMBER 4, 2018 : FM

Preprint typeset using IATEX style emulateapj v. 11/10/09

\title{
BL LAC CANDIDATES FOR TEV OBSERVATIONS
}

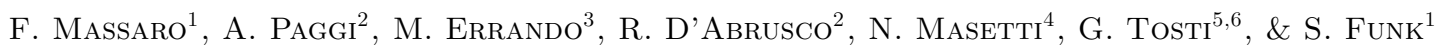 \\ version September 4, $2018: \mathrm{fm}$
}

\begin{abstract}
BL Lac objects are the most numerous class of extragalactic TeV-detected sources. One of the biggest difficulties in investigating their $\mathrm{TeV}$ emission resides in their limited number, since only 47 BL Lacs are known as TeV emitters. In this paper, we propose new criteria to select TeV BL Lac candidates based on the infrared (IR) and X-ray observations. We apply our selection criteria to the BL Lac objects listed in the ROMA-BZCAT catalog so identifying 41 potential TeV emitters. We then consider a search over a more extended sample combining the ROSAT bright source catalog and the WISE all-sky survey revealing 54 additional candidates for TeV observations. Our investigation also led to a tentative classification of 16 unidentified X-ray sources as BL Lac candidates. This analysis provides new interesting BL Lac targets for future observations with ground based Cherenkov telescopes.

Subject headings: galaxies: active - galaxies: BL Lacertae objects - X-rays: galaxies: individual: radiation mechanisms: non-thermal
\end{abstract}

\section{INTRODUCTION}

BL Lac objects are characterized by very peculiar properties with respect to other classes of active galactic nuclei $(\mathrm{AGNs})$. They are compact, core dominated radio sources, many of them exhibiting superluminal motion and showing rapid and large-amplitude flux variability from radio up to $\mathrm{TeV}$ energies, and significant radio to optical polarization (e.g., Blandford \& Rees 1978; Urry \& Padovani 1995). Their spectral energy distribution (SED) exhibits two main components: the low energy one peaking in the infrared-X-ray energy range, and the second one dominated by $\gamma$-rays. Their optical spectra appear to be featureless or with very weak absorption lines (Stoke et al. 1991; Stickel et al. 1991; Laurent-Muehleisen et al. 1999).

According to Padovani \& Giommi (1995) BL Lacs can be classified as "Low-frequency peaked BL Lacs" (LBLs) and "High-frequency peaked BL Lacs" (HBLs), depending on whether their broadband radio-to-X-ray spectral index is larger than or smaller than 0.75 , respectively.

At very high energies (i.e., $E>100 \mathrm{GeV}$ ) BL Lac objects, and in particular, HBLs, constitute the largest known population of $\mathrm{TeV}$ extragalactic sources, detected by ground based Cherenkov telescopes as HESS, MAGIC and VERITAS. In the following, we refer to the HBLs detected at $\mathrm{TeV}$ energies as TBLs while we indicate the HBL candidates for TeV observations as TBCs.

Recently, using the WISE point source catalog, which mapped the sky in four different bands centered at 3.4, 4.6, 12, and $22 \mu \mathrm{m}$ (Wright et al. 2010;

\footnotetext{
${ }^{1}$ SLAC National Laboratory and Kavli Institute for Particle Astrophysics and Cosmology, 2575 Sand Hill Road, Menlo Park, CA 94025, USA

${ }^{2}$ Smithsonian Astrophysical Observatory, 60 Garden Street, Cambridge, MA 02138, USA

${ }^{3}$ Department of Physics and Astronomy, Barnard College, Columbia University, New York, NY 10027, USA

${ }^{4}$ INAF - Istituto di Astrofisica Spaziale e Fisica Cosmica di Bologna, via Gobetti 101, 40129, Bologna, Italy

${ }^{5}$ Dipartimento di Fisica, Università degli Studi di Perugia, 06123 Perugia, Italy
}

Cutri et al. 2012), we discovered that $\gamma$-ray emitting blazars occupy a distinct region in the two-dimensional color-color diagrams, which is well separated from other extragalactic sources whose IR emission is dominated by thermal radiation ("the WISE Gamma-ray Strip", Massaro et al. 2011a; D'Abrusco et al. 2012) and we have developed a method for identifying $\gamma$-ray blazar candidates by studying the WISE three-dimensional IR color space using the WISE Fermi Blazar Sample (i.e., "locus", see D'Abrusco et al. 2013). This discovery constitutes the basis of our selection criterion for the TBCs.

In this paper, we combine IR and X-ray archival data available in literature to build a criterion useful to find new TBCs. We use the X-ray observations performed with ROSAT along with those from the Wide Infrared Survey Explorer (WISE) satellite (Wright et al. 2010).

This paper is organized as follows: in $\S 2$ we investigate the IR properties of the BL Lacs already detected at $\mathrm{TeV}$ energies introducing the " $\Phi_{X I R}$ parameter" to distinguish between LBLs and HBLs. In $\S 3$ we outline our criterion to identify TBCs and apply it to the BL Lacs listed in the ROMA-BZCAT6 (e.g., Massaro et al. 2011b) and comparisons with selection criteria previously published are presented in $\S[4 . \S 5$ is dedicated to the all-sky search of new TBL candidates using the combination of the ROSAT bright source catalog (Voges et al. 1999) and the WISE all-sky survey (Wright et al. 2010) and $\S$ 6 is devoted to our summary and conclusions.

WISE magnitudes are in the Vega system and we use cgs units for our numerical results unless stated otherwise. We assume a flat cosmology with $H_{0}=72 \mathrm{~km}$ $\mathrm{s}^{-1} \mathrm{Mpc}^{-1}, \Omega_{M}=0.26$ and $\Omega_{\Lambda}=0.74$ (Dunkley et al. 2009). Spectral indices, $\alpha$, are defined by flux density, $\mathrm{S}_{\nu} \propto \nu^{-\alpha}$. Frequent acronyms are listed in Table 1.

\section{TEV BL LAC OBJECTS}

According to the online catalog of TeV-emitting

${ }^{6}$ http://www.asdc.asi.it/bzcat/ 
TABLE 1

LIST OF ACRONYMS.

\begin{tabular}{|lc|}
\hline Name & Acronym \\
\hline High Frequency Peaked BL Lac & HBL \\
Low Frequency Peaked BL Lac & LBL \\
HBL detected at TeV energies & TBL \\
HBL candidate for TeV observations & TBC \\
\hline
\end{tabular}

$\gamma$-ray sources (TeVCat)7, the number of sources classified as BL Lac objects in the ROMABZCAT (Massaro et al. 2009; Massaro et al. 2010; Massaro et al. 2011b) and detected at TeV energies is 42, as of December 2012; these TeV BL Lac objects are listed in Table 2 together with their salient parameters. They have a unique WISE counterpart detected at least at $3.4,4.6$ and $12 \mu \mathrm{m}$ within a radius of $3^{\prime \prime} .3$ from the ROMA-BZCAT positions (see D'Abrusco et al. 2013, for more details about the ROMA-BZCAT - WISE positional associations). They also have a radio counterpart and are detected in the X-ray band by ROSAT (Voges et al. 1999) as reported in the ROMA-BZCAT (Massaro et al. 2011b) with the only exception of MAGICJ2001+435. Thirty-seven of them are also detected in $\gamma$ rays between $30 \mathrm{MeV}$ and $100 \mathrm{GeV}$ as reported in the Fermi-LAT second source Catalog (2FGL; Nolan et al. 2012) and in the second Fermi-LAT AGN catalog (2LAC; Ackermann et al. 2011).

\subsection{X-ray-to-infrared flux ratio: $\Phi_{X I R}$}

Maselli et al. (2010a) defined the ratio $\Phi_{X R}$ between the ROSAT X-ray flux $F_{X}$ and the radio flux density $S_{1.4}$ (at $1.4 \mathrm{GHz}$ ), computed using the values reported in the ROMA-BZCAT, to distinguish between HBLs (i.e., $\Phi_{X R} \geq 0.1$ ) and LBLs (i.e., $\Phi_{X R}<0.1$ ). However, this distinction cannot be easily extended all-sky because it does need radio observations at $1.4 \mathrm{GHz}$ which are not always available. To avoid this problem we define a new parameter to distinguish between the two subclasses of BL Lac objects based on the IR observations of WISE. It is worth noting that among the BL Lac objects the HBLs are the most detected at $\mathrm{TeV}$ energies.

For all the $\mathrm{TeV}$ BL Lac objects listed in Table 2 we computed $\Phi_{X I R}$, defined as the ratio between the ROSAT X-ray flux $F_{X}(0.1-2.4 \mathrm{keV})$ and the integrated IR flux $F_{I R}$ between 3.4 and $12 \mu \mathrm{m}$, both in units of $10^{-12} \mathrm{erg} \mathrm{cm}^{-2} \mathrm{~s}^{-1}$. This parameter is used to distinguish between HBLs and LBLs instead of $\Phi_{X R}$. We note that sources with $\Phi_{X I R}>0.1$ in Table 2 have a $\Phi_{X R}>0.08$ in agreement with the previous classification (Maselli et al. 2010a; Maselli et al. 2010b). We then consider a new classification, labeling as HBLs those having $\Phi_{X I R}>0.1$ while indicating as LBLs those with $\Phi_{X I R}<0.1$. An additional justification on the choice of $\Phi_{X I R}$ to classify BL Lacs is given in Appendix on the basis of their spectral shape.

\subsection{TBL sample selection}

We define a clean sample of $33 \mathrm{HBLs} \mathrm{TeV}$ detected (i.e., TBLs) out of $42 \mathrm{TeV}$ sources including only:

- having $\Phi_{X I R}>0.1$;

7 http://tevcat.uchicago.edu/
- with WISE magnitudes lower than 13.32 mag, $12.64 \mathrm{mag}$ and $10.76 \mathrm{mag}$ at $3.4 \mu \mathrm{m}, 4.6 \mu \mathrm{m}$ and $12 \mu \mathrm{m}$, respectively;

- with IR colors between $0.22 \mathrm{mag}<[3.4]-[4.6]<0.86$ mag, $1.60 \mathrm{mag}<[4.6]-[12]<2.32 \mathrm{mag}$.

The above criterion of TBLs, not only based on the $\Phi_{X R}$ ratio, permits to select bright IR sources having the first SED peak between the UV and the X-rays. The minimum X-ray and IR fluxes of the resulting sample are $2.45 \cdot 10^{-12} \mathrm{erg} \mathrm{cm}^{-2} \mathrm{~s}^{-1}$ and $9.47 \cdot 10^{-13} \mathrm{erg} \mathrm{cm}^{-2} \mathrm{~s}^{-1}$, respectively.

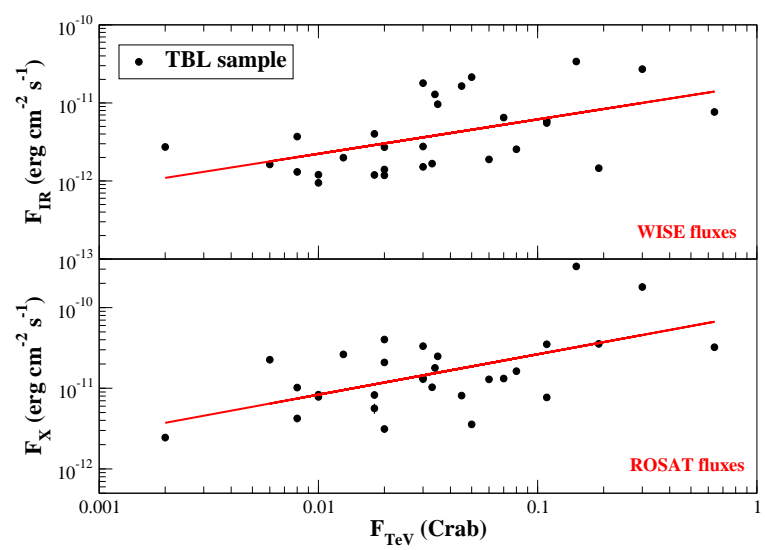

FIG. 1.- The correlations between the IR (upper panel) and the $\mathrm{X}$-ray (lower panel) fluxes with the TeV flux, reported in the WISE ROSAT and TeVCat catalogs, respectively (see also Table 2). Regression lines are shown in red (see Section 2.2 for more details).

As shown in Figure 1, there is a hint of a correlation between the IR and TeV fluxes for the TBLs whose measurements were available in TeVCat (see also Table 2), with a correlation coefficient of 0.51 . This suggests a good match between WISE and the TeV observations. As expected there is also a trend between the ROSAT and the TeV fluxes, with a correlation coefficient of 0.58 .

\subsection{Infrared colors of TBLs}

Recently, we discovered that the $\gamma$-ray BL Lac objects lie in a region (i.e., the WISE Gamma-ray Strip) of the [3.4]-[4.6]-[12] $\mu \mathrm{m}$ color-color diagram well differentiated from that occupied by generic IR sources (Massaro et al. 2011a). In particular, TBLs are more concentrated near the tail of the WISE Gamma-ray Strip. In Figure 2 we show the IR colors of TBLs and those of $\gamma$-ray BL Lacs detected by Fermi in the 2LAC CLEAN sample that belong to the WISE Gamma-ray Strip (D'Abrusco et al. 2013).

We calculated a linear regression in the [3.4]-[4.6]-[12] $\mu \mathrm{m}$ color-color plot for the 33 selected TBLs as shown in Figure 2, We then define the $\delta$ parameter according to the equation:

$$
\delta=\left|D \cdot D_{\max }^{-1}\right|
$$

where $D$ is the distance between the IR colors of each source in the [3.4]-[4.6]-[12] $\mu \mathrm{m}$ color-color diagram and 

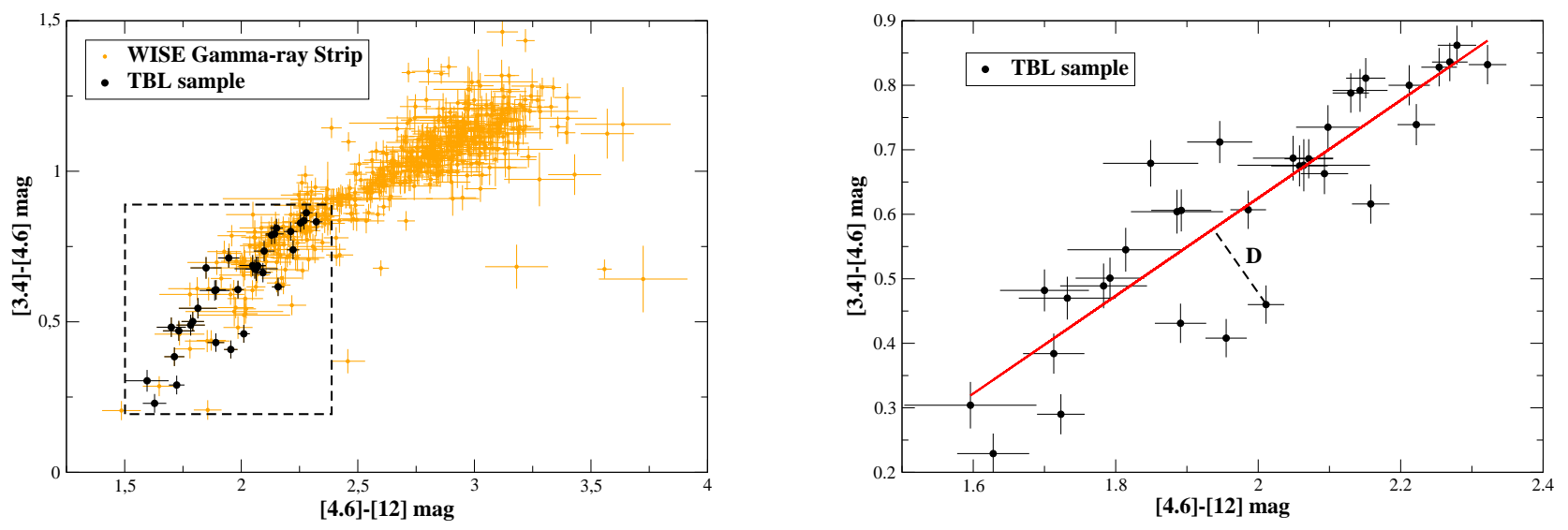

FIG. 2.- Left panel: the [3.4]-[4.6]-[12] $\mu \mathrm{m}$ color-color plot for the 33 TBLs selected (black circles) overlaid to the $\gamma$-ray emitting blazars associated with WISE source that constitute the WISE Gamma-ray Strip (see Massaro et al. 2011a; D'Abrusco et al. 2012; D'Abrusco et al. 2013, for more details). The black dashed box indicates the subregion of the WISE Gamma-ray Strip considered in our TBC selection. Right panel: the [3.4]-[4.6]-[12] $\mu \mathrm{m}$ color-color plot for the 33 TBLs selected (black circles). The red line corresponds to the regression line evaluated while the dashed line indicates the distance $D$ between a source and the regression line as described in $\S 2.3$

the regression line, and $D_{\max }$ is the maximum value evaluated only for the selected TBLs (i.e., $0.116 \mathrm{mag}$ ). All the TBLs have therefore a value of delta $; 1.0$ by definition. We verified the residuals of the points in the [3.4]-[4.6]-[12] $\mu \mathrm{m}$ color-color plot with respect to the regression line using a runs test and we found that they are randomly distributed at $97 \%$ level of confidence 8 .

Finally, we note that $\delta=0.28$ corresponds to the $68 \%$ level of confidence (i.e., $1 \sigma$ ) with respect to the regression line and consequently the choice of $\delta=1$ implies that all the TBLs lie within $3.5 \sigma$.

\section{TBL CANDIDATES SELECTED FROM THE ROMA-BZCAT CATALOG}

On the basis of the combined IR and X-ray properties of TBLs (see $\S 2$ ), we outline the following criteria to select TBL candidates (TBCs). Our selection of TBCs includes all sources that fulfill all following criteria:

1. classified as BL Lac (i.e., BZB) according to the ROMA-BZCAT catalog;

2. have a WISE counterpart within $3.3^{\prime \prime}$ from the ROMA-BZCAT position, detected with Vega magnitudes smaller than $13.318,12.642$ and 10.760 at $3.4 \mu \mathrm{m}, 4.6 \mu \mathrm{m}$ and $12 \mu \mathrm{m}$, respectively;

3. have IR colors similar to those of TBLs defined by: $0.22 \mathrm{mag}<[3.4]-[4.6]<0.86 \mathrm{mag}, 1.60 \mathrm{mag}<[4.6]$ $[12]<2.32$ mag, respectively;

4. have values of the parameter $\delta<1$, according to the definition proposed in $\S 2.3$

5. have X-ray fluxes larger than the minimum value observed for TBLs (i.e., $2.45 \cdot 10^{-12} \mathrm{erg} \mathrm{cm}^{-2} \mathrm{~s}^{-1}$ ).

8 The runs test is a non-parametric statistical test that verifies the hypothesis that the elements of the sequence are mutually independent and it could be applied in combination with a regression analysis to check that residuals are randomly distributed as expected in a Gaussian statistic.
The requirement on the X-ray and IR fluxes ensures that the selected sources will be above ROSAT and WISE sensitivity thresholds.

We apply our selection to the BL Lac objects that belong to the ROMA-BZCAT (Massaro et al. 2009; Massaro et al. 2010; Massaro et al. 2011b).

For the blazars listed in the ROMA-BZCAT we found 41 TBCs that meet our criteria. All these TBCs are detected by Fermi in the $30 \mathrm{MeV}-300 \mathrm{GeV}$ energy range with only five exceptions: BZB J0056-0936, BZB J0214+5144, BZB J0809+3455, BZB J1215+0732 and BZB J1445-0326, in particular, about $~ 90 \%$ of them show hard $\gamma$-ray spectra (i.e., $\gamma$-ray photon index $\Gamma<2$ ). Their complete list can be found in Table 3, where we report their ROMA-BZCAT name, that of their WISE counterpart, the redshift if known, the ROSAT X-ray flux corrected for the Galactic absorption (Kalberla et al. 2005), the Fermi $\gamma$-ray spectral index, the IR WISE colors together with the IR flux in the 3.4$12 \mu \mathrm{m}$ band and the value of $\Phi_{X I R}$.

Finally, we note that, all the TBCs selected from the ROMA-BZCAT lie within $3 \sigma$ level of confidence of the regression line (see Section 2.3 ), with the only exceptions of BZB J0214+5144 and BZB J1445-0326.

\section{COMPARISON WITH PREVIOUS SELECTIONS}

Several attempts to select BL Lac candidates for TeV observations have been carried out in the last decade, with particular attention to HBLs as in our analysis. Selection of source candidates has typically relied on the availability of source catalogs at lower frequencies that could reveal properties characteristic of VHE emitters. For instance, blazar candidates for VHE observations were typically selected from cata$\operatorname{logs}$ of hard X-ray sources (e.g., Stecker et al. 1996; Donato et al. 2001) or objects that had a particular combination of radio, optical and X-ray energy densities (Costamante \& Ghisellini 2002).

In particular, Costamante \& Ghisellini (2002) proposed a selection of BL Lac candidates for TeV observa- 
TABLE 2

The Complete list of TeV Detected BL Lac objects $(00-24 \mathrm{HH})$.

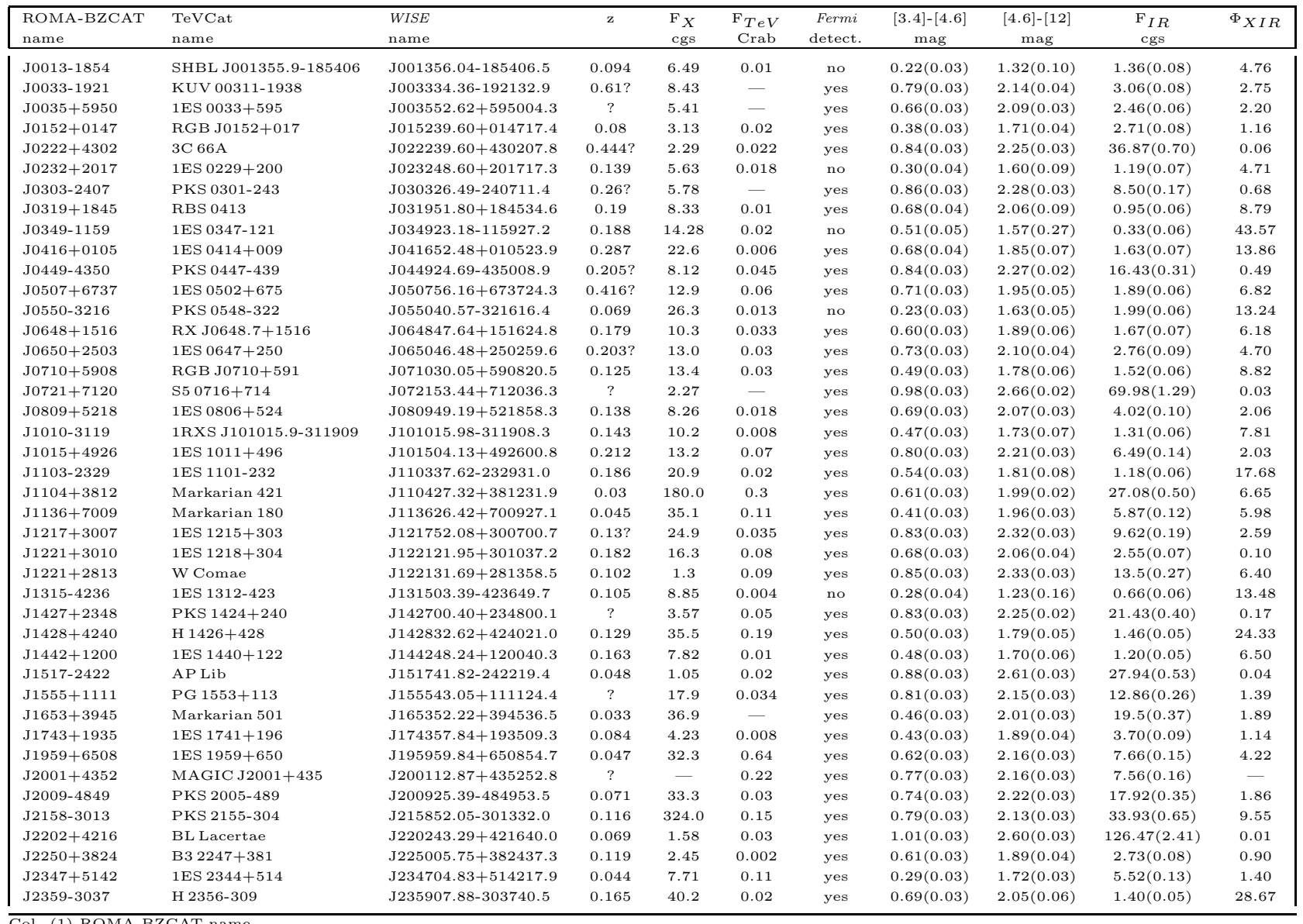

Col. (2) TeVCat name.

Col. (4) ROMA-BZCAT redshift: ? = unknown, number? = uncertain

Col. (5) ROSAT X-ray flux in the $0.1-2.4 \mathrm{keV}$ energy range in units of $10^{-12} \mathrm{erg} \mathrm{cm}^{-2} \mathrm{~s}^{-1}$.

Col. (5) ROSAT X-ray flux in the $0.1-2.4 \mathrm{keV}$ energy

Col. (6) archival TeV flux as reported on the TeVCa

Col. (7) Fermi detection as reported in the 2FGL

theses are $1 \sigma$ uncertainties.

Col. (10) WISE IR flux in the $3.4-12 \mu \mathrm{m}$ energy range in units of $10^{-12} \mathrm{erg} \mathrm{cm}^{-2} \mathrm{~s}^{-1}$

Col. (11) $\Phi_{X I R}$ defined according to $\S$

tions, not only restricted to the HBLs as in the present analysis. Their selection was mainly based on a fitting procedure of the broadband SEDs of a sample of BL Lacs compiled from literature with a homogeneous synchrotron self Compton model and calculating the expected TeV flux. They conclude that TeV BL Lac candidates are primarily selected to be bright both in X-rays and radio bands, as generally occurs for HBLs. Tavecchio et al. (2010) also proposed a selection of BL Lac candidates for $\mathrm{TeV}$ observations on the basis of the $\gamma$ ray properties, such as hard $\gamma$-ray spectra, of the Fermi sources detected in its first three months of operation. Recently, Massaro et al. (2011c) also outlined a criterion to select only TBCs, mainly based on the X-ray spectral curvature and applied to the HBLs detected in the major X-ray surveys as Einstein Slew Survey (e.g., Elvis et al.1992). This was the first criterion developed on the basis of the BL Lac spectral shape observed in a restricted energy range.

In comparison with the analyses cited above, 8 out of 41 of the TBCs selected were also present in their lists. We note that 2 sources appear in Stecker et al.
(1996) list of candidates and 8 in that of Costamante \& Ghisellini (2002), with 2 objects, BZB J0326+0225 and BZB J1728+5013, present in both selections. Three of our sources appear as TeV candidates in the Tavecchio et al. (2010) selection: BZB J0109+1816, BZB J0136+3905 and BZB J1058+5628; the last source also in Costamante \& Ghisellini (2002).

In Massaro et al. (2011c) we also propose a X-ray based selection and BZB J0326+0225, BZB J1136+6737, BZB J1417+2543, and BZB J1728+5013, were deeply investigated and selected as TBCs on the basis of their Xray spectral curvature. In particular, BZB J1728+5013 is present in all the previous selections with the only exception of Tavecchio et al. (2010), making it the most promising TBC.

The main difference between our method and the previous selections is that it is based on the IR rather than on the radio flux density and that was built on the basis of the peculiar IR colors of the known TBLs (i.e., a surrogate of the IR spectral shape), an information that was not used in all the previous selections. It is also worth noting that all $\sim 90 \%$ BL Lacs of the ROMA-BZCAT 
TABLE 3

The COMPlete List of TBCs SELECTED From the ROMA-BZCAT (00 - 24 HH).

\begin{tabular}{|c|c|c|c|c|c|c|c|c|c|c|}
\hline $\begin{array}{l}\text { ROMA-BZCAT } \\
\text { name }\end{array}$ & $\begin{array}{l}\text { WISE } \\
\text { name }\end{array}$ & $\bar{z}$ & $\begin{array}{l}\mathrm{F}_{X} \\
\mathrm{cgs}\end{array}$ & $\Gamma$ & $\begin{array}{c}{[3.4]-[4.6]} \\
\mathrm{mag}\end{array}$ & $\begin{array}{c}4.6]-[12] \\
\mathrm{mag}\end{array}$ & $\begin{array}{l}\mathrm{F}_{I R} \\
\text { cgs }\end{array}$ & $\Phi_{X I R}$ & Sel. & \\
\hline BZB J0035+1515 & J003514.71+151504.2 & ? & 3.02 & 1.62 & $0.78(0.03)$ & $2.20(0.06)$ & $1.63(0.07)$ & 1.86 & - & \\
\hline BZB J0056-0936 & J005620.06-093630.6 & 0.103 & 4.20 & - & $0.29(0.03)$ & $1.75(0.05)$ & $2.52(0.08)$ & 1.67 & - & \\
\hline BZB J0109+1816 & J010908.17+181607.7 & 0.145 & 4.51 & 1.99 & $0.81(0.03)$ & $2.28(0.05)$ & $1.87(0.06)$ & 2.42 & $\mathrm{~T}$ & \\
\hline BZB J0136+3905* & J013632.59+390559.2 & $?$ & 9.60 & 1.69 & $0.79(0.03)$ & $2.11(0.03)$ & $4.01(0.09)$ & 2.39 & $\mathrm{C}, \mathrm{T}$ & \\
\hline BZB J0209-5229 & J020921.60-522922.7 & $?$ & 7.98 & 1.91 & $0.61(0.03)$ & $1.97(0.05)$ & $1.58(0.05)$ & 5.06 & - & \\
\hline BZB J0214+5144 & J021417.94+514451.9 & 0.049 & 4.58 & - & $0.29(0.03)$ & $1.77(0.04)$ & $3.48(0.09)$ & 1.31 & M & \\
\hline BZB J0238-3116 & J023832.47-311657.9 & ? & 5.14 & 1.85 & $0.64(0.03)$ & $1.91(0.04)$ & $1.96(0.06)$ & 2.62 & - & \\
\hline BZB J0316-2607 & J031614.93-260757.2 & 0.443 & 3.05 & 1.87 & $0.75(0.03)$ & $2.14(0.04)$ & $1.34(0.04)$ & 2.27 & - & \\
\hline BZB J0325-1646 & J032541.09-164616.8 & 0.291 & 27.2 & 1.97 & $0.70(0.04)$ & $2.02(0.07)$ & $1.10(0.05)$ & 24.77 & - & \\
\hline BZB J0326+0225 & J032613.94+022514.7 & 0.147 & 12.0 & 2.06 & $0.58(0.04)$ & $2.02(0.08)$ & $1.19(0.06)$ & 10.07 & $\mathrm{C}, \mathrm{M}, \mathrm{S}$ & \\
\hline BZB J0505+0415 & J050534.76+041554.5 & $0.027 ?$ & 3.07 & 2.15 & $0.60(0.04)$ & $2.03(0.08)$ & $1.02(0.06)$ & 3.02 & - & \\
\hline BZB J0536-3343 & J053629.06-334302.5 & ? & 4.84 & 2.39 & $0.68(0.03)$ & $2.04(0.05)$ & $1.31(0.05)$ & 3.70 & - & \\
\hline BZB J0543-5532 & J054357.21-553207.5 & ? & 9.04 & 1.74 & $0.69(0.03)$ & $2.00(0.04)$ & $1.57(0.04)$ & 5.75 & - & \\
\hline BZB J0805+7534 & J080526.63+753424.9 & 0.121 & 3.66 & 1.68 & $0.54(0.03)$ & $2.02(0.04)$ & $1.94(0.06)$ & 1.89 & - & \\
\hline BZB J0809+3455 & J080938.91+345537.3 & 0.083 & 4.07 & - & $0.33(0.03)$ & $1.69(0.07)$ & $1.69(0.07)$ & 2.40 & - & \\
\hline BZB J0913-2103 & J091300.22-210321.0 & 0.198 & 12.4 & 1.94 & $0.62(0.03)$ & $2.06(0.04)$ & $2.57(0.07)$ & 4.83 & - & \\
\hline BZB J0915+2933 & J091552.40+293324.0 & ? & 6.25 & 1.87 & $0.76(0.03)$ & $2.24(0.04)$ & $3.31(0.09)$ & 1.89 & - & \\
\hline BZB J1023-4336 & $\mathrm{J} 102356.20-433601.5$ & ? & 13.4 & 1.82 & $0.78(0.03)$ & $2.06(0.04)$ & $2.16(0.06)$ & 6.19 & - & \\
\hline BZB J1058+5628* & $\mathrm{J} 105837.73+562811.3$ & 0.143 & 3.13 & 1.93 & $0.80(0.03)$ & $2.28(0.03)$ & $6.07(0.13)$ & 0.52 & $\mathrm{~T}$ & \\
\hline BZB J1117+2014 & J111706.26+201407.5 & 0.138 & 33.6 & 1.70 & $0.61(0.03)$ & $2.05(0.05)$ & $1.98(0.07)$ & 16.99 & $\mathrm{C}$ & \\
\hline BZB J1120+4212 & $\mathrm{J} 112048.06+421212.6$ & $0.124 ?$ & 7.81 & 1.61 & $0.70(0.03)$ & $1.98(0.07)$ & $1.18(0.05)$ & 6.62 & - & \\
\hline BZB J1136+6737 & $\mathrm{J} 113630.10+673704.4$ & 0.136 & 14.8 & 1.68 & $0.44(0.03)$ & $1.87(0.06)$ & $1.16(0.05)$ & 12.71 & $\mathrm{C}$ & \\
\hline BZB J1215+0732 & $\mathrm{J} 121510.98+073204.7$ & 0.136 & 3.27 & - & $0.42(0.04)$ & $1.76(0.08)$ & $1.19(0.06)$ & 2.75 & - & \\
\hline BZB J1241-1455 & J124149.40-145558.4 & $?$ & 8.37 & 1.98 & $0.68(0.03)$ & $2.04(0.06)$ & $1.51(0.06)$ & 5.55 & - & \\
\hline BZB J1243+3627* & $\mathrm{J} 124312.74+362744.0$ & $?$ & 10.0 & 1.70 & $0.78(0.03)$ & $2.20(0.03)$ & $3.97(0.09)$ & 2.52 & - & \\
\hline BZB J1248+5820 & $\mathrm{J} 124818.79+582028.8$ & $?$ & 3.99 & 1.95 & $0.86(0.03)$ & $2.29(0.03)$ & $7.59(0.15)$ & 0.53 & - & \\
\hline BZB J1417+2543 & $\mathrm{J} 141756.67+254325.9$ & 0.237 & 15.3 & 1.98 & $0.53(0.03)$ & $2.02(0.05)$ & $1.16(0.04)$ & 13.20 & $\mathrm{C}, \mathrm{M}$ & \\
\hline BZB J1439+3932 & $\mathrm{J} 143917.48+393242.8$ & 0.344 & 11.1 & 1.69 & $0.72(0.03)$ & $2.10(0.04)$ & $1.99(0.05)$ & 5.57 & - & \\
\hline BZB J1443-3908 & J144357.20-390839.9 & $0.065 ?$ & 6.56 & 1.77 & $0.73(0.03)$ & $2.16(0.03)$ & $4.48(0.11)$ & 1.46 & - & \\
\hline BZB J1445-0326 & J144506.24-032612.5 & ? & 3.21 & - & $0.69(0.03)$ & $1.86(0.08)$ & $0.92(0.05)$ & 3.48 & - & \\
\hline BZB J1448+3608 & $\mathrm{J} 144800.59+360831.2$ & $?$ & 4.62 & 1.89 & $0.77(0.03)$ & $2.13(0.04)$ & $1.55(0.04)$ & 2.99 & - & \\
\hline BZB J1501+2238 & J150101.83+223806.3 & 0.235 & 4.02 & 1.77 & $0.83(0.03)$ & $2.28(0.03)$ & $6.15(0.12)$ & 0.65 & - & \\
\hline BZB J1540+8155 & $\mathrm{J} 154015.90+815505.6$ & $?$ & 5.77 & 1.48 & $0.69(0.03)$ & $2.00(0.04)$ & $1.40(0.04)$ & 4.12 & $\mathrm{C}$ & \\
\hline BZB J1548-2251 & J154849.76-225102.5 & ? & 6.21 & 1.93 & $0.70(0.03)$ & $2.11(0.05)$ & $2.16(0.08)$ & 2.88 & - & \\
\hline BZB J1725+1152 & $\mathrm{J} 172504.34+115215.5$ & $?$ & 11.5 & 1.93 & $0.81(0.03)$ & $2.16(0.03)$ & $4.53(0.11)$ & 2.54 & $\mathrm{C}$ & \\
\hline BZB J1728+5013 & $\mathrm{J} 172818.63+501310.5$ & 0.055 & 20.4 & 1.83 & $0.62(0.03)$ & $2.18(0.03)$ & $3.13(0.07)$ & 6.52 & $\mathrm{C}, \mathrm{M}, \mathrm{S}$ & \\
\hline BZB J1917-1921 & J191744.82-192131.5 & 0.137 & 2.86 & 1.91 & $0.84(0.03)$ & $2.27(0.03)$ & $6.16(0.15)$ & 0.46 & - & \\
\hline BZB J2221-5225 & $\mathrm{J} 222129.30-522527.6$ & ? & 5.43 & 2.06 & $0.72(0.03)$ & $2.17(0.05)$ & $1.42(0.04)$ & 3.83 & - & \\
\hline BZB J2323+4210 & $\mathrm{J} 232352.07+421058.6$ & $0.059 ?$ & 2.69 & 1.88 & $0.77(0.03)$ & $2.28(0.07)$ & $1.09(0.05)$ & 2.47 & - & \\
\hline BZB J2324-4040 & $\mathrm{J} 232444.65-404049.3$ & $?$ & 14.6 & 1.81 & $0.75(0.03)$ & $2.06(0.03)$ & $4.77(0.11)$ & 3.06 & - & \\
\hline BZB J2340+8015 & $\mathrm{J} 234054.23+801515.9$ & 0.274 & 3.39 & 1.87 & $0.75(0.03)$ & $2.12(0.04)$ & $2.48(0.06)$ & 1.37 & - & \\
\hline $\begin{array}{ll}\text { Col. (1) ROMA-BZC } \\
\text { Col. (2) WISE name. } \\
\text { Col. (3) ROMA-BZC }\end{array}$ & redshift: ? = unkno & numb & & & & & & $\mathrm{S} \sqrt{\mathrm{A}}$ & & 2, see also Section 6 . \\
\hline $\begin{array}{l}\text { Col. (4) ROSAT X-ra } \\
\text { Col. (5) 2FGL } \gamma \text {-ray } \\
\text { Cols. (6,7) IR colors }\end{array}$ & $\begin{array}{l}\text { lux in the } 0.1-2.4 \text { ke } \\
\text { oton index } \Gamma \text {. } \\
\mathrm{m} \text { WISE. Values in p }\end{array}$ & 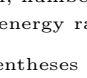 & & & $-12 \mathrm{erg}$ & $-2-1$ & . & & & (Kalberla et al. 2005). \\
\hline
\end{tabular}

Costamante \& Ghisellini (2002; - C), Tavecchio et al. (2010; - T), and Massaro et al. (2012c; - M) (see $\S$ 田 for more details).

that met our criteria are also detected in the $\gamma$-rays by Fermi, while this was not a requirement for our selection.

\section{ALL-SKY INFRARED SEARCH OF TBL CANDIDATES}

We extended our search of TBCs beyond the ROMABZCAT catalog by considering X-ray sources from the ROSAT bright source catalog (Voges et al. 1999) with a counterpart in the WISE all-sky survey (Wright et al. 2010) and adopting less restrictive criteria than the one previously described in $\S 3$.

We considered all the IR sources detected by WISE that lie within the positional uncertainty of an X-ray source in the ROSAT bright source catalog. Then, we selected only IR sources with WISE magnitudes smaller than $13.32 \mathrm{mag}, 12.64 \mathrm{mag}$ and $10.76 \mathrm{mag}$ at $3.4 \mu \mathrm{m}$, $4.6 \mu \mathrm{m}$ and $12 \mu \mathrm{m}$, respectively, IR colors between 0.23 $\operatorname{mag}<[3.4]-[4.6]<0.86 \mathrm{mag} 1.60 \mathrm{mag}<[4.6]-[12]<2.32$ mag and $\delta<1$ (see $\S 2.3$ and $\S 3$ for more details). This criterion corresponds to a less restrictive selection than the one previously proposed, because it is not based on the X-ray flux nor on the ratio $\Phi_{X I R}$.

The ROSAT bright source catalog lists 18811 X-ray sources all-sky, however only 189 of them met the criteria outlined above. All of them are unique associations be- tween the ROSAT and the WISE all-sky surveys. Moreover, out of the 189 selected sources, 93 are associated to sources listed in the ROMA-BZCAT catalog. These were excluded from our extended TBL candidate list to avoid redundancy in the selections.

For the remaining 96 sources, we performed a multifrequency analysis to select the most reliable TBCs. We searched in the following major radio, IR, optical databases as well as in the NASA Extragalactic Database (NED) 9 for any possible counterpart within $3^{\prime \prime} .3$ to verify if additional information can confirm their BL Lac nature.

For the radio surveys we searched in the catalogs of the NRAO VLA Sky Survey (NVSS; Condon et al. 1998), the VLA Faint Images of the Radio Sky at Twenty-Centimeters (FIRST; Becker et al. 1995; White et al. 1997), the Sydney University Molonglo Sky Survey (SUMSS; Mauch et al. 2003) and the The Australia Telescope $20 \mathrm{GHz}$ Survey (AT20G; Murphy et al. 2010) surveys; for the IR we compare our list only with the Two Micron All Sky

${ }^{9}$ http://ned.ipac.caltech.edu/ 
Survey (2MASS; Skrutskie et al. 2006) since each WISE source is already associated with the closest 2MASS source by default in the WISE catalog (see Cutri et al. 2012, for more details). Then, we also searched for optical counterparts, with possible spectra available, in the Sloan Digital Sky Survey (SDSS; e.g. Adelman et al. 2008; Paris et al. 2012) and in the Six-degree-Field Galaxy Redshift Survey (6dFGS; Jones et al. 2004; Jones et al. 2009); in the hard X-rays within the $2^{\text {nd }}$ Palermo BAT catalog (2PBC; Cusumano et al. 2010) and if associated with Fermi source in the 2FGL (Nolan et al. 2012). We also searched the USNO-B Catalog (Monet et al. 2003) for the optical counterparts within $3^{\prime \prime} .3$ and we report the magnitude in the $\mathrm{R}$ band in Table 4. Our final list has been also compared with the recent WISE-2MASSROSAT selection of active galaxies proposed by Edelson \& Malkan (2012).

On the basis of our multifrequency investigation we selected 54 TBCs out of the 96 remaining sources selected combining the ROSAT all-sky survey with the WISE observations. All these new 54 TBCs are listed in Table 4 together with the results of our multifrequency analysis and their salient parameters as the IR WISE colors and, if present, the 2FGL association (e.g., Ackermann et al. 2011; Nolan et al. 2012).

A large fraction of our TBCs $(\sim 55 \%)$ have a clear radio counterpart in one of the major radio surveys as occurs for all the BL Lacs that belong to the WISE Gammaray strip. In particular, 21 out of 54 sources have a radio counterpart in the NVSS, 4 in the SUMSS, one in both radio surveys while only 2 objects have a correspondence in the FIRST. All our 54 TBCs are detected in the 2MASS catalog and they are also detected by WISE at $22 \mu \mathrm{m}$ with two exceptions. Optical spectra are available in literature for 5 TBCs listed in Table 4 all classified as BL Lac objects, thus no optical spectroscopic observations are necessary to confirm their nature. Then, 21 out of 54 TBCs have been observed by the 6dFGS and 4 by the SDSS. At high energies only one source has been detected by Swift-BAT hard X-ray survey while 14 out of 54 objects have been associated or lie within the positional uncertainty region of a Fermi source listed in the 2FGL. It is worth noting that 14 out of 54 TBCs are associated with Fermi sources as listed in the 2FGL catalog and in the 2LAC catalogs, being classified as AGNs of uncertain type (Nolan et al. 2012; Ackermann et al. 2011). In particular, 1RXS J083158.1-180828, 1RXS J130421.2435308 and 1RXS J204745.9-024609 are the only three sources that belong to our final list of TBCs selected combining WISE and ROSAT all-sky catalogs that were also selected as active galaxies of uncertain nature by Edelson \& Malkan (2012).

Moreover, 16 X-ray sources out of 54 TBCs were previously unidentified in the ROSAT all-sky catalog (Voges et al. 1999) (i.e., without a counterpart assigned at lower energies). The existence of an IR WISE counterpart in the WISE catalog, with similar colors than those of $\gamma$-ray BL Lacs suggests that these could be potential BL Lac candidates. We note that none of these 54 TBCs is listed in the Sedentary Survey of extreme HBLs (Giommi et al. 2005), thus highlighting that our method is successful to select BL Lacs without including any criteria based on the radio observations.

Forty-two out of 189 IR-X-ray sources we selected have multiwavelength archival observations that indicate they are not BL Lacs. This suggest that a contamination of $\sim 22 \%$ of non-BL Lac objects could be present in our selected sample. We will be able to make a more accurate estimate of the contamination once all the optical spectroscopic information will be available for our sample.

Finally, we note that all the TBCs selected from the ROSAT bright source catalog with a counterpart in the WISE all-sky survey lie within $3 \sigma$ level of confidence of the regression line (see Section 2.3), with the only exceptions of 5 sources, namely: 1RXS J072812.1+671821, 1RXS J132908.3+295018, 1RXS J180219.5-245157, 1RXS J183821.0-602519 and 1RXS J193320.3+072616.

\section{SUMMARY AND CONCLUSIONS}

Previous studies based on the WISE all-sky survey have revealed that the IR spectral shape of high frequency peaked BL Lacs detected at $\mathrm{TeV}$ energies (TBLs) can be successfully used to associate $\gamma$-ray BL Lacs (e.g., Massaro et al. 2011a; Massaro et al. 2012b; D'Abrusco et al. 2013). In this paper, we extended the same technique to search for high frequency peaked BL Lacs that could be candidates for future TeV observations (i.e., TBCs), by selecting sources with similar IR and X-ray properties of the known TBLs.

Known TBLs populate a subregion of the WISE Gamma-ray Strip (Massaro et al. 2012a; D'Abrusco et al. 2013), defined in the [3.4]-[4.6][12] $\mu \mathrm{m} \mathrm{IR} \mathrm{color-color} \mathrm{space.} \mathrm{Then,} \mathrm{on} \mathrm{the} \mathrm{basis} \mathrm{of}$ their IR and the X-ray emission, we identify 41 TBCs among the BL Lacs listed in the ROMA-BZCAT catalog (Massaro et al. 2009; Massaro et al. 2011b).

A comparison between our list of TBCs, chosen out of the ROMA-BZCAT catalog, with previous selections (Stecker et al. 1996; Costamante \& Ghisellini 2002; Tavecchio et al. 2010; $\quad$ Massaro et al. 2011c) finds good agreement (see $\S$ 4). Our new criteria, mainly based on the IR colors, a surrogate of the spectral shape of the low energy component for the BL Lac objects, is not based on radio or $\gamma$-ray data. Moreover, our IR selection was built only in the 2-dimensional [3.4]-[4.6]-[12] $\mu \mathrm{m}$ color-color diagram, while all our previous selections of $\gamma$-ray blazar candidates, mostly developed to associate unidentified gamma-ray sources (Massaro et al. 2012b; D'Abrusco et al. 2013), required the IR detection in all four WISE bands. All the BL Lacs of the ROMA-BZCAT that met our criteria are also detected in the $\gamma$-ray band by Fermi, while this was not a requirement for the selection discussed in this paper.

We note that VERITAS observations have been performed for 3 of our TBCs selected within the ROMABZCAT: BZB J0136+3905, BZB J1058+5628 and BZB $\mathrm{J} 1243+3627$ (Aliu et al. 2012). However, as discussed in Aliu et al. (2012), both BZB J0136+3905 and BZB $\mathrm{J} 1243+3627$ do not have a redshift estimate, indicating that their non TeV-detection could be due the absorption of high energy photons by the extragalactic background light (Franceschini et al. 2008). On the other hand, BZB $\mathrm{J} 1058+5628$ was found variable in the $\gamma$-rays by Fermi during the same period of the VERITAS observations. 
Thus, the non-detection at $\mathrm{TeV}$ energies of these three sources does not affect our selection that can only be verified with additional $\mathrm{TeV}$ observations.

We conducted an extended search based on less restrictive criteria based on the combination of the WISE and ROSAT observations to search for new TBCs with IR properties similar to those of the TBLs in the Xray sky. We found additional 54 sources that could be considered TBCs with pending confirmation of their BL Lac nature with follow-up optical spectroscopy. We also note that 16 TBCs out of $54 \mathrm{X}$ ray sources were previously unidentified in the ROSAT bright source catalog (Voges et al. 1999); then we provide the first association with a low energy counterpart correspondent to our TBCs selected on the basis of the IR WISE colors. We note that only 21 out of a total of 95 TBCs (i.e., 41 selected in the ROMA-BZCAT and 54 in the all-sky search) have a reliable redshift determination. The TBC with the highest redshift is BZB J0316-2607 at $\mathrm{z}=0.443$ closer than the most distant $\mathrm{TeV}$ source: $3 \mathrm{C} 279$ at $\mathrm{z}=0.5362$ (e.g., Errando et al. 2008).

Our investigation provides new targets to plan observations with ground based Cherenkov telescopes such as HESS, MAGIC and VERITAS or in the near future with CTA.

We thank the anonymous referee for useful comments that improved the presentation of our work. We are grateful to $\mathrm{R}$. Mukherjee for her valuable comments and suggestions that improved the manuscript as well as to S. Digel, P. Giommi, D. Harris and J. Grindlay for their helpful discussions. The work is supported by the NASA grants NNX12AO97G. R. D'Abrusco grate- fully acknowledges the financial support of the US Virtual Astronomical Observatory, which is sponsored by the National Science Foundation and the National Aeronautics and Space Administration. The work by G. Tosti is supported by the ASI/INAF contract I/005/12/0. M. Errando acknowledges support from the NASA grant NNX12AJ30G. TOPCAT10 (Taylor 2005) was used extensively in this work for the preparation and manipulation of the tabular data and the images. Part of this work is based on archival data, software or on-line services provided by the ASI Science Data Center. This research has made use of data obtained from the High Energy Astrophysics Science Archive Research Center (HEASARC) provided by NASA's Goddard Space Flight Center; the SIMBAD database operated at CDS, Strasbourg, France; the NASA/IPAC Extragalactic Database (NED) operated by the Jet Propulsion Laboratory, California Institute of Technology, under contract with the National Aeronautics and Space Administration. Part of this work is based on the NVSS (NRAO VLA Sky Survey); The National Radio Astronomy Observatory is operated by Associated Universities, Inc., under contract with the National Science Foundation. This publication makes use of data products from the Two Micron All Sky Survey, which is a joint project of the University of Massachusetts and the Infrared Processing and Analysis Center/California Institute of Technology, funded by the National Aeronautics and Space Administration and the National Science Foundation. This publication makes use of data products from the Wide-field Infrared Survey Explorer, which is a joint project of the University of California, Los Angeles, and the Jet Propulsion Laboratory/California Institute of Technology, funded by the National Aeronautics and Space Administration.

\section{REFERENCES}

Abdo, A. A., et al. 2009, Astroparticle Physics, 32, 193

Abdo, A. A. et al. 2010 ApJS 188405

Ackermann, M. et al. 2011 ApJ, 743, 171

Ackermann, M. et al. 2012 ApJ, 753, 83

Adelman-McCarthy, J., Agueros, M.A., Allam, S.S., et al. 2008, ApJS, 175, 297

Ali, E. et al. 2012 ApJ, 759, 102

Becker, R. H., White, R. L., Helfand, D. J.1995 ApJ, 450, 559

Blandford, R. D., Rees, M. J., 1978b PhyS, 17, 265

Blandford, R. D. \& Königl, A.1979 ApJ, 232, 34

Condon, J. J., Cotton, W. D., Greisen, E. W., Yin, Q. F., Perley, R. A., Taylor, G. B., \& Broderick, J. J. 1998, AJ, 115, 1693

Costamante, L. \& Ghisellini, G. 2002 A\&A, 384, 56

Cusumano, G. et al. 2010 A\&A, 524A, 64

Cutri et al. 2012 wise.rept, $1 \mathrm{C}$

D'Abrusco, R., Massaro, F., Ajello, M., Grindlay, J. E., Smith, Howard A. \& Tosti, G. 2012 ApJ, 748, 68

D'Abrusco, R., Massaro, F., Paggi, A., Masetti, N., Giroletti, M., Tosti, G. et al. 2013 ApJS submitted

Donato, D., Ghisellini, G., Tagliaferri, G. \& Fossati, G., 2001, A\&A, 375, 739

Edelson, R. \& Malkan, M. 2012 ApJ, 751, 52

Elvis, M., Plummer, D., Schachter, J., Fabbiano, G. 1992 ApJS, 80,257

Errando, M., Bock, R., Kranich, D., Lorenz, E., Majumdar, P., Mariotti, M., Mazin, D., Prandini, E. et al. 2008 AIPC, 1085, 423

Franceschini, A., Rodighiero, G., Vaccari, M. 2008 A\&A, 487, 837

Giommi, P. et al. 2005, A\&A, 434, 385

Giommi, P. et al. 2007 A\&A, 468, 571

10 http://www.star.bris.ac.uk/ mbt/topcat/
Giommi, P., Colafrancesco, S., Padovani, P., Gasparrini, D.,

Cavazzuti, E., Cutini, S. 2009, A\&A, 508, 107

Giommi, P. et al. 2012a A\&A, 541A, 160

Giommi, P., Padovani, P., Polenta, G., Turriziani, S., D'Elia, V.,

Piranomonte, S. 2012b MNRAS, 420, 2899

González-Nuevo, J. et al. 2010 A\&A, 518, L38

Hartman, R.C. et al., 1999 ApJS 123

Howard, W. E. III, Dennis, T. R., Maran, S. P.; Aller, H. D. 1965 ApJS, 10, 331

Kalberla, P.M.W., Burton, W.B., Hartmann, D., 2005, A\&A, 440, 775

Impey, C. D. \& Neugebauer, G. 1988 AJ, 95, 307

Jones, H. D. et al. 2004 MNRAS, 355, 747

Jones, H. D. et al. 2009 MNRAS, 399, 683

Landau, R., Golish, B., Jones, T. J., et al. 1986, ApJ, 308, L78

Laurent-Muehleisen, S. A., Kollgaard, R. I., Feigelson, E. D., Brinkmann, W., Siebert, J. 1999 ApJ, 525, 127

Maselli, A., Massaro, E., Nesci, R., Sclavi, S., Rossi, C., Giommi, P. 2010a A\&A, 512A, 74

Maselli, A., Cusumano, G., Massaro, E., La Parola, V., Segreto, A., Sbarufatti, B. 2010b A\&A, 520A, 47

Massaro, E., Perri, M., Giommi, P., et al. 2004, A\&A, 422, 103

Massaro, F. et al. 2008a A\&A, 489, 1047

Massaro, F., Tramacere, A., Cavaliere, A., Perri, M., Giommi, P. 2008b A\&A, 478, 395

Massaro, E., Giommi, P., Leto, C., Marchegiani, P., Maselli, A., Perri, M., Piranomonte, S., Sclavi, S. 2009 A\&A, 495, 691

Massaro, E., Giommi, P., Leto, C., Marchegiani, P., Maselli, A., Perri, M., Piranomonte, S., Sclavi, S. 2010

http://arxiv.org/abs/1006.0922

Massaro, F., D'Abrusco, R., Ajello, M., Grindlay, J. E. \& Smith, H. A. 2011 ApJ, 740L, 48 
TABLE 4

TBCS ALL-SKY SELECTED FROM THE ROSAT - WISE ALL-SKY SURVEYS $(00-24 \mathrm{HH})$

\begin{tabular}{|c|c|c|c|c|c|c|c|c|}
\hline $\begin{array}{l}\text { ROSAT } \\
\text { name }\end{array}$ & $\begin{array}{l}W I S E \\
\text { name }\end{array}$ & $\begin{array}{l}\text { other } \\
\text { name }\end{array}$ & $\begin{array}{c}{[3.4]-[4.6]} \\
\text { mag }\end{array}$ & $\begin{array}{c}{[4.6]-[12]} \\
\mathrm{mag}\end{array}$ & $\begin{array}{c}{[12]-[22]} \\
\mathrm{mag}\end{array}$ & $\begin{array}{c}\mathrm{R} \\
\mathrm{mag}\end{array}$ & notes & $\mathrm{z}$ \\
\hline J001541.3+555141 & J001540.13+555144.7 & NVSS J001540+555144 & $0.59(0.03)$ & $1.93(0.05)$ & $2.14(0.15)$ & 16.07 & $\mathrm{~N}, \mathrm{M}$ & $?$ \\
\hline J002159.2-514028 & J002200.08-514024.2 & SUMSS J002159-514026 & $0.81(0.03)$ & $2.23(0.04)$ & $1.87(0.14)$ & 15.94 & $\mathrm{~S}, \mathrm{M}, 6, \mathrm{v}, \mathrm{f}$ & $?$ \\
\hline J002922.4+505159 & J002921.68+505159.0 & & $0.86(0.03)$ & $2.22(0.05)$ & $2.12(0.15)$ & 16.61 & M,UNID & $?$ \\
\hline J005447.2-245532 & J005446.74-245529.0 & NVSS J005446-245529 & $0.74(0.04)$ & $1.95(0.08)$ & $<1.82$ & 17.08 & $\mathrm{~N}, \mathrm{M}, 6, \mathrm{f}, \mathrm{BL}$ & $?$ \\
\hline J010325.9+533721 & J010325.95+533713.3 & NVSS J010326+533712 & $0.45(0.04)$ & $1.91(0.06)$ & $1.74(0.26)$ & 14.94 & $\mathrm{~N}, \mathrm{M}, \mathrm{f}$ & $?$ \\
\hline J013445.2-043017 & J013445.62-043012.9 & 6dF J0134455-043013 & $0.74(0.03)$ & $2.21(0.03)$ & $2.19(0.04)$ & 13.41 & $\mathrm{~N}, \mathrm{M}, 6, \mathrm{v}$ & $?$ \\
\hline J014100.4-675332 & J014100.45-675327.2 & 6dF J0141003-675328 & $0.60(0.03)$ & $1.85(0.05)$ & $0.77(0.51)$ & 15.83 & $\mathrm{M}, 6, \mathrm{v}$ & $?$ \\
\hline J021652.4-663644 & J021650.85-663642.5 & SUMSS J021650-663643 & $0.73(0.03)$ & $2.12(0.05)$ & $2.18(0.13)$ & 17.14 & $\mathrm{~S}, \mathrm{M}, 6, \mathrm{f}$ & $?$ \\
\hline $\mathrm{J} 024215.2+053037$ & J024214.63+053036.0 & NVSS J024214+053042 & $0.77(0.03)$ & $2.32(0.03)$ & $1.82(0.05)$ & 12.03 & $\mathrm{~N}, \mathrm{M}, \mathrm{v}, \mathrm{B}$ & $?$ \\
\hline J032220.5-305929 & J032220.09-305933.9 & 6dF J0322201-305934 & $0.79(0.03)$ & $2.17(0.04)$ & $1.97(0.15)$ & 16.00 & $\mathrm{M}, 6, \mathrm{v}$ & $?$ \\
\hline J033118.2-615532 & J033118.46-615528.8 & 6dF J0331185-615529 & $0.66(0.03)$ & $2.14(0.04)$ & $1.84(0.17)$ & 16.20 & $\mathrm{M}, 6$ & $?$ \\
\hline J033913.4-173553 & J033913.70-173600.6 & NVSS J033913-173600 & $0.32(0.03)$ & $1.72(0.04)$ & $2.00(0.16)$ & 10.98 & $\mathrm{~N}, \mathrm{~A}, \mathrm{M}, 6, \mathrm{f}$ & $0.0656 ?$ \\
\hline J034203.8-211428 & J034203.71-211439.3 & NVSS J034203-211449 & $0.68(0.03)$ & $2.24(0.03)$ & $1.82(0.02)$ & 9.27 & $\mathrm{~N}, \mathrm{M}, 6$ & $?$ \\
\hline $\mathrm{J} 043917.9+224802$ & J043917.42+224753.3 & & $0.46(0.03)$ & $1.80(0.03)$ & $1.49(0.03)$ & 13.03 & $\mathrm{M}$ & $?$ \\
\hline J045142.3-034834 & J045141.51-034833.6 & NVSS J045141-034834 & $0.55(0.03)$ & $2.00(0.03)$ & $1.96(0.04)$ & 8.25 & $\mathrm{~N}, \mathrm{M}, 6, \mathrm{~B}$ & $?$ \\
\hline J051952.0-512347 & J051952.79-512338.0 & & $0.73(0.03)$ & $2.30(0.03)$ & $2.13(0.06)$ & 14.72 & $\mathrm{M}$ & $?$ \\
\hline J062040.0+264339 & J062040.05+264331.9 & NVSS J062040+264331 & $0.51(0.03)$ & $1.88(0.08)$ & $2.23(0.25)$ & 15.80 & $\mathrm{~N}, \mathrm{M}$ & $?$ \\
\hline J062221.4-260537 & J062222.06-260544.6 & NVSS J062222-260544 & $0.82(0.03)$ & $2.29(0.04)$ & $2.10(0.11)$ & 16.94 & $\mathrm{~N}, \mathrm{M}, 6, \mathrm{f}, \mathrm{BL}$ & $?$ \\
\hline J063923.6+010231 & J063923.53+010231.2 & & $0.79(0.04)$ & $2.28(0.06)$ & $2.55(0.12)$ & 16.01 & M,UNID & $?$ \\
\hline J064007.4-125316 & J064007.19-125315.0 & NVSS J064007-125315 & $0.46(0.03)$ & $1.74(0.04)$ & $1.95(0.15)$ & 13.69 & $\mathrm{~N}, \mathrm{~A}, \mathrm{M}, 6$ & $?$ \\
\hline J065610.6+460538 & J065609.67+460541.5 & & $0.80(0.03)$ & $2.29(0.04)$ & $2.22(0.10)$ & 15.17 & M,UNID & $?$ \\
\hline J070912.3-152708 & J070912.51-152703.6 & NVSS J070912-152701 & $0.48(0.03)$ & $1.63(0.05)$ & $2.35(0.14)$ & 15.54 & $\mathrm{~N}, \mathrm{M}$ & $?$ \\
\hline J072259.5-073131 & J072259.68-073135.0 & NVSS J072259-073135 & $0.75(0.04)$ & $1.98(0.06)$ & $2.10(0.24)$ & 16.77 & $\mathrm{~N}, \mathrm{M}$ & $?$ \\
\hline J072812.1+671821 & J072812.88+671814.7 & & $0.78(0.03)$ & $1.97(0.06)$ & $2.04(0.21)$ & 16.56 & M,UNID & $?$ \\
\hline J073048.2-660212 & J073049.52-660218.9 & & $0.47(0.03)$ & $1.88(0.04)$ & $2.23(0.09)$ & 14.95 & M,UNID & $?$ \\
\hline J073143.9-470009 & J073144.11-470008.4 & & $0.54(0.08)$ & $1.92(0.06)$ & $2.06(0.08)$ & - & M,UNID & $?$ \\
\hline J082705.9-070841 & J082706.17-070845.9 & NVSS J082706-070846 & $0.64(0.03)$ & $1.97(0.04)$ & $1.64(0.17)$ & 14.75 & $\mathrm{~N}, \mathrm{M}, 6, \mathrm{BL}$ & $0.12 ?$ \\
\hline J083158.1-180828 & J083158.37-180835.2 & 6dF J0831584-180835 & $0.82(0.03)$ & $2.24(0.04)$ & $2.29(0.08)$ & 15.74 & $\mathrm{M}, 6$ & $?$ \\
\hline J094709.2-254056 & J094709.52-254100.0 & NVSS J094709-254100 & $0.73(0.04)$ & $2.17(0.06)$ & $2.06(0.23)$ & 16.71 & $\mathrm{~N}, \mathrm{M}, 6, \mathrm{f}$ & $?$ \\
\hline $\mathrm{J} 130421.2-435308$ & $\mathrm{~J} 130421.01-435310.2$ & SUMSS J130420-435308 & $0.86(0.03)$ & $2.27(0.03)$ & $1.93(0.05)$ & 16.10 & $\mathrm{~S}, \mathrm{M}, \mathrm{f}, \mathrm{v}$ & $?$ \\
\hline $\mathrm{J} 130737.8-425940$ & J130737.98-425938.9 & SUMSS J130737-425940 & $0.74(0.03)$ & $2.06(0.03)$ & $1.90(0.08)$ & 15.58 & $\mathrm{~S}, \mathrm{M}, 6, \mathrm{f}, \mathrm{v}$ & $?$ \\
\hline $\mathrm{J} 132452.1+213559$ & $\mathrm{~J} 132451.92+213548.7$ & SDSSJ132451.91+213548.8 & $0.67(0.03)$ & $2.17(0.04)$ & $2.04(0.13)$ & 14.67 & $\mathrm{~F}, \mathrm{M}, \mathrm{s}$ & $?$ \\
\hline $\mathrm{J} 132908.3+295018$ & $\mathrm{~J} 132908.84+295024.2$ & SDSSJ132908.83+295024.2 & $0.61(0.03)$ & $2.21(0.06)$ & $1.94(0.21)$ & 14.75 & $\mathrm{M}, \mathrm{s}$ & $?$ \\
\hline $\mathrm{J} 134751.3+283639$ & $\mathrm{~J} 134751.55+283631.5$ & SDSSJ134751.52+283632.3 & $0.36(0.03)$ & $1.73(0.06)$ & $2.65(0.15)$ & 14.47 & $\mathrm{~F}, \mathrm{M}, \mathrm{s}$ & ? \\
\hline J140906.7-451714 & J140907.20-451715.8 & $6 \mathrm{dF}$ J1409074-451716 & $0.56(0.03)$ & $1.84(0.04)$ & $1.81(0.15)$ & 14.25 & $\mathrm{M}, 6, \mathrm{v}$ & $?$ \\
\hline J150554.3-694935 & J150555.68-694932.6 & & $0.85(0.03)$ & $2.16(0.05)$ & $2.33(0.10)$ & 17.55 & M,UNID & $?$ \\
\hline J153548.6-295904 & J153548.53-295855.5 & $6 \mathrm{dF}$ J1535486-295854 & $0.34(0.03)$ & $1.73(0.03)$ & $1.98(0.04)$ & 13.38 & $\mathrm{M}, 6$ & $?$ \\
\hline J154513.6-341733 & J154512.84-341730.6 & & $0.75(0.06)$ & $2.08(0.03)$ & $2.04(0.02)$ & 9.63 & M,UNID & $?$ \\
\hline $\mathrm{J} 170034.7-273807$ & J170034.97-273804.4 & & $0.45(0.05)$ & $1.65(0.03)$ & $2.14(0.03)$ & 10.40 & M,UNID & $?$ \\
\hline $\mathrm{J} 180219.5-245157$ & J180219.45-245154.3 & & $0.51(0.03)$ & $1.61(0.02)$ & $2.29(0.02)$ & - & $\mathrm{M}, \mathrm{v}, \mathrm{UNID}$ & $?$ \\
\hline $\mathrm{J} 180925.6+204130$ & $\mathrm{~J} 180925.43+204131.2$ & NVSS J180925+204131 & $0.80(0.04)$ & $2.12(0.06)$ & $1.52(0.33)$ & 16.49 & $\mathrm{~N}, \mathrm{M}, \mathrm{f}$ & $?$ \\
\hline J182022.7-101104 & J182022.75-101113.4 & & $0.33(0.04)$ & $1.63(0.03)$ & $2.12(0.02)$ & 10.12 & M,UNID & $?$ \\
\hline J182339.2-345412 & J182338.59-345412.0 & NVSS J182338-345412 & $0.70(0.04)$ & $2.07(0.04)$ & $1.92(0.13)$ & - & $\mathrm{N}, \mathrm{A}, \mathrm{M}, \mathrm{f}$ & $?$ \\
\hline J183821.0-602519 & J183820.64-602522.4 & & $0.33(0.03)$ & $1.83(0.07)$ & $2.26(0.19)$ & 13.11 & M,UNID & $?$ \\
\hline $\mathrm{J} 184121.8+290932$ & $\mathrm{~J} 184121.73+290940.9$ & NVSS J184121+290945 & $0.61(0.04)$ & $2.09(0.06)$ & $1.92(0.26)$ & 16.66 & $\mathrm{~N}, \mathrm{M}$ & $?$ \\
\hline $\mathrm{J} 192503.1+504315$ & $\mathrm{~J} 192502.18+504313.8$ & & $0.69(0.03)$ & $2.20(0.03)$ & $2.35(0.04)$ & 14.68 & M,UNID & ? \\
\hline $\mathrm{J} 192649.5+615445$ & $\mathrm{~J} 192649.89+615442.4$ & NVSS J192649+615441 & $0.79(0.03)$ & $2.18(0.04)$ & $1.88(0.13)$ & 17.20 & $\mathrm{~N}, \mathrm{M}, \mathrm{f}$ & ? \\
\hline $\mathrm{J} 193320.3+072616$ & $\mathrm{~J} 193320.30+072621.9$ & NVSS J193320+072619 & $0.85(0.05)$ & $2.07(0.08)$ & $1.90(0.34)$ & 16.60 & $\mathrm{~N}, \mathrm{M}, \mathrm{UNID}$ & ? \\
\hline $\mathrm{J} 195020.5+331419$ & $\mathrm{~J} 195019.72+331416.2$ & & $0.85(0.03)$ & $2.17(0.03)$ & $1.75(0.12)$ & 15.82 & M,UNID & ? \\
\hline J195815.6-301119 & J195814.91-301111.5 & NVSS J195814-301112 & $0.42(0.03)$ & $1.86(0.07)$ & $2.12(0.25)$ & 13.97 & $\mathrm{~N}, \mathrm{~S}, \mathrm{M}, \mathrm{s}, 6, \mathrm{f}, \mathrm{BL}$ & ? \\
\hline J204149.8-373346 & J204150.23-373339.8 & $6 \mathrm{dF}$ J2041502-373340 & $0.31(0.03)$ & $1.63(0.08)$ & $<2.25$ & 13.62 & $\mathrm{M}, 6, \mathrm{BL}$ & 0.0986 \\
\hline J204745.9-024609 & $\mathrm{J} 204745.80-024604.1$ & NVSS J204745-024605 & $0.85(0.03)$ & $2.32(0.04)$ & $1.98(0.09)$ & 15.01 & $\mathrm{~N}, \mathrm{~A}, \mathrm{M}, 6$ & $?$ \\
\hline $\mathrm{J} 224427.7+440135$ & $\mathrm{~J} 224427.24+440137.4$ & & $0.72(0.03)$ & $2.32(0.03)$ & $2.12(0.04)$ & 13.43 & M,UNID & $?$ \\
\hline $\mathrm{J} 224753.3+441321$ & $\mathrm{~J} 224753.19+441315.6$ & NVSS J224753+441317 & $0.69(0.03)$ & $2.13(0.06)$ & $1.94(0.22)$ & 16.75 & $\mathrm{~N}, \mathrm{M}, \mathrm{f}$ & $?$ \\
\hline
\end{tabular}

Col. (1) ROSAT name.

Col. (2) WISE name.

Col. (3) Other name if present in literature and in the following order: NVSS, SDSS, AT20G, NED

Cols. ( $4,5,6)$ IR colors from WISE. Values in parentheses are $1 \sigma$ uncertainties

Col. (7) Notes: $\mathrm{N}=$ NVSS, $\mathrm{F}=\mathrm{FIRST}, \mathrm{S}=\mathrm{SUMSS}, \mathrm{A}=\mathrm{AT} 20 \mathrm{G}, \mathrm{M}=2 \mathrm{MASS}, \mathrm{s}=\mathrm{SDSS}$ dr9, $6=6 \mathrm{dFGS}, \mathrm{x}=$ XMM-Newton or Chandra, $\mathrm{X}=\mathrm{ROSAT} ; \mathrm{B}=$ Swift-BAT; $\mathrm{f}=$ Fermi

$\mathrm{BL}=\mathrm{BL}$ Lac (optical spectra available in Jones et al. 2009); $\mathrm{v}=$ variability in WISE (var_flag $>5$ in at least one band); UNID=ROSAT unidentified X-ray source.
Col. (10) Redshift: ? = unknown, number? = uncertain.

Massaro, E., Giommi, P., Leto, C., Marchegiani, P., Maselli, A., Perri, M., Piranomonte, S., 2011 "Multifrequency Catalogue of Blazars (3rd Edition)", 2011a ARACNE Editrice, Rome, Italy Massaro, F., Paggi, A., Elvis, M., Cavaliere, A. 2011 ApJ, 739, 73

Massaro, F., D'Abrusco, R., Tosti, G., Ajello, M., Gasparrini, D., Grindlay, J. E. \& Smith, Howard A. 2012b ApJ, 750, 138

Massaro, F., D'Abrusco, R., Tosti, G., Ajello, M., Paggi, A., Gasparrini, 2012c ApJ, 752, 61

Massaro, F. et al. 2013 ApJS in preparation

Mauch, T., Murphy, T., Buttery, H. J., Curran, J., Hunstead, R. W., Piestrzynski, B., Robertson, J. G., Sadler, E. M. 2003 MNRAS, 342, 1117

Monet, D. G. et al. 2003 AJ, 125, 984

Mukherjee, R. et al., 1997 ApJ, 490, 116

Murphy, T. et al. 2010 MNRAS, 402, 2403

Nolan et al. 2012 ApJS, 199, 31

Paris, I. et al. 2012 A\&A, 548A, 66

Schneider et al. 2007, AJ, 134, 102

Skrutskie, M. F. et al. 2006, AJ, 131, 1163
Stickel, M., Padovani, P., Urry, C. M., Fried, J. W., Kuehr, H. 1991 ApJ, 374, 431

Stocke et al. 1991, ApJS, 76, 813

$\mathrm{Su}$, M. \& Finkbeiner, D. P. 2012 ApJ submitted

http://arxiv.org/abs/1207.7060v1

Stecker, F. W.; de Jager, O. C.; Salamon, M. H. 1996 ApJ, 473L, 75

Urry, C. M., \& Padovani, P. 1995, PASP, 107, 803

Tavecchio, F., Ghisellini, G., Ghirlanda, G., Foschini, L., Maraschi, L. 2010 MNRAS, 401,1570

Taylor, M. B. 2005, ASP Conf. Ser., 347, 29

$2008 \mathrm{RPPh}, 71 \mathrm{k} 6901$

Tramacere, A., Massaro, F., Cavaliere, A., 2007, A\&A, 466, 521

Voges, W. et al. 1999 A\&A, 349, 389

White, R. L., Becker, R. H. Helfand, D. J., Gregg, M. D. et al. 1997 ApJ, 475, 479

Wright, E. L., et al. 2010 AJ, 140, 1868

Zechlin, H.-S., Fernandes, M. V., Elsasser, D., Horns, D. 2012 A\&A, 538A, 93 


\section{APPENDIX}

To further justify the classification scheme proposed in Section 2.1 based on $\Phi_{X I R}$, we assumed a broadband description of the BL Lac spectra, from the IR to the X-rays, in the form of a log-parabola (e.g., Howard et al. 1965; Landau et al. 1986), expressed as:

$$
S_{\nu}=\frac{S_{p}}{\nu} \cdot\left(\frac{\nu}{\nu_{p}}\right)^{-b \log \left(\nu / \nu_{p}\right)} \operatorname{erg~cm}^{-2} s^{-1} H z^{-1}
$$

where $\nu_{p}$ is the SED peak frequency, $S_{p}$ the SED peak flux at $\nu_{p}$, and $b$ the spectral curvature (see Massaro et al. 2004; Tramacere et al. 2007, for more details). We computed the ratio $\Phi_{X I R}$ as function of the peak frequency $\nu_{p}$ for different values of $b$ as shown in Figure 3 . Thus for values of $\nu_{p}$ larger than $10^{15} \mathrm{~Hz}$, as generally seen for HBLs, $\Phi_{X I R}$ is systematically larger than 0.1 (see Figure 3). Values of spectral curvature used in Figure 3 are those typically observed in BL Lac objects (Massaro et al. 2008a; ; Massaro et al. 2008b; Massaro et al. 2011b).

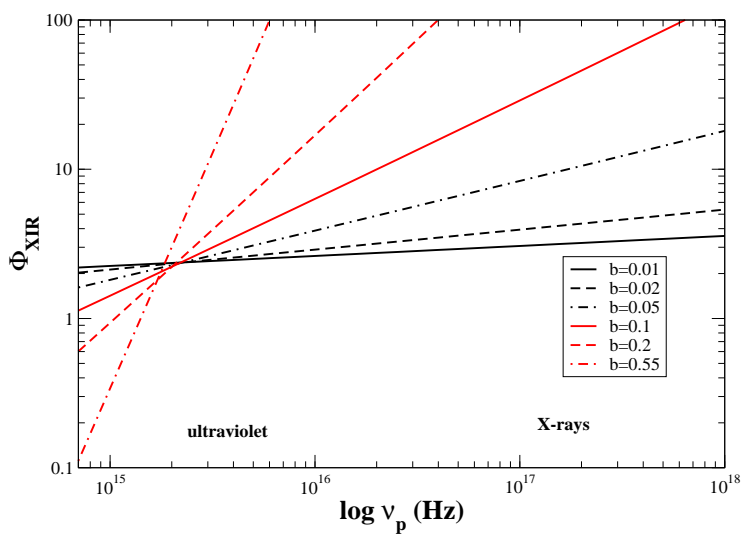

FIG. 3.- The values of $\Phi_{X I R}$ as function of the peak frequency $\nu_{p}$ of the log-parabolic function (see Eq. 1) assumed as simple representation of the low energy component of the BL Lac SED. The different lines correspond to different values of the spectral curvature $b$, typical of BL Lac objects (e.g., Massaro et al. 2008a; ; Massaro et al. 2008b). 